\title{
Chronological Review of Endoscopic Indices in Inflammatory Bowel Disease
}

\author{
Joon Seop Lee ${ }^{1}$, Eun Soo Kim ${ }^{1}$ and Won Moon ${ }^{2}$ \\ ${ }^{1}$ Department of Internal Medicine, Kyungpook National University School of Medicine, Daegu, ${ }^{2}$ Department of Internal Medicine, Kosin \\ University College of Medicine, Busan, Korea
}

Inflammatory bowel disease (IBD) is considered a chronic condition characterized by mucosal or transmural inflammation in the gastrointestinal tract. Endoscopic diagnosis and surveillance in patients with IBD have become crucial. In addition, endoscopy is a useful modality in estimation and evaluation of the disease, treatment results, and efficacy of treatment delivery and surveillance. In relation to these aspects, endoscopic disease activity has been commonly estimated in clinical practices and trials.

At present, many endoscopic indices of ulcerative colitis have been introduced, including the Truelove and Witts Endoscopy Index, Baron Index, Powell-Tuck Index, Sutherland Index, Mayo Clinic Endoscopic Sub-Score, Rachmilewitz Index, Modified Baron Index, Endoscopic Activity Index, Ulcerative Colitis Endoscopic Index of Severity, Ulcerative Colitis Colonoscopic Index of Severity, and Modified Mayo Endoscopic Score. Endoscopic indices have been also suggested for Crohn's disease, such as the Crohn's Disease Endoscopic Index of Severity, Simple Endoscopic Score for Crohn's Disease, and Rutgeerts Postoperative Endoscopic Index. However, most endoscopic indices have not been validated owing to the complexity of their parameters and inter-observer variations. Therefore, a chronological approach for understanding the various endoscopic indices relating to IBD is needed to improve the management.

Clin Endosc 2019;52:129-136

Key Words: Colitis, ulcerative; Crohn disease; Endoscopic index; Colonoscopy

\section{INTRODUCTION}

Inflammatory bowel disease (IBD) is considered a chronic phenomenon that induces mucosal or transmural inflammation in the gastrointestinal tract. Endoscopy has a critical role in the treatment of patients with IBD by estimating the severity of disease activity in the intestinal mucosa and the efficacy of therapeutic agents. ${ }^{1}$ As a result, endoscopic diagnosis and healing in patients with IBD have become crucial. ${ }^{2}$ Because ulcerative colitis (UC) presents mucosal lesions involving

\section{Received: February 22, 2018 Revised: April 30, 2018}

Accepted: May 2, 2018

Correspondence: Won Moon

Department of Internal Medicine, Kosin University College of Medicine, 262 Gamcheon-ro, Seo-gu, Busan 49267, Korea

Tel: +82-51-990-5207, Fax: +82-51-990-5055, E-mail: moonone70@hanmail.net ORCID: https://orcid.org/0000-0002-3963-8680

(cc) This is an Open Access article distributed under the terms of the Creative Commons Attribution Non-Commercial License (http://creativecommons.org/ licenses/by-nc/3.0) which permits unrestricted non-commercial use, distribution, and reproduction in any medium, provided the original work is properly cited. the large intestine, the role of endoscopy is also integral. By contrast, inflammation in Crohn's disease (CD) commonly manifests as transmural inflammation and involves the entire intestine; therefore, affected areas are not accessible by an endoscope. ${ }^{3}$ However, endoscopy has integral roles in diagnosing the involvement and severity of diseases, e.g., estimation of treatment efficacy and delivery and cancer surveillance. ${ }^{4}$ Furthermore, since the introduction of new therapies using anti-tumor necrosis factor drugs, the management of patients with IBD has changed substantially. Therefore, endoscopic disease activity has been commonly estimated in clinical practices and trials.

In addition to endoscopic estimation, a new concept of managing the involved intestines in patients with IBD has emerged, i.e., mucosal healing (MH). In IBD, the term $\mathrm{MH}$ is described on the basis of endoscopic assessment and is usually considered to reflect healing of the mucosal lesions. ${ }^{5} \mathrm{MH}$ provides an objective estimation of medical treatment and alleviates the placebo effect associated with the clinical indices used 
previously. Accumulating evidence suggests that $\mathrm{MH}$ may modify the natural course of IBD. ${ }^{6}$ At present, $\mathrm{MH}$ is defined as resolution of all ulcerations and histologic inflammation. Not presenting endoscopic $\mathrm{MH}$ can increase the incidence of colorectal tumors in UC. In addition, MH has decreased the frequency of surgery and rates of hospitalization in $\mathrm{CD}^{7}$ In these regards, $\mathrm{MH}$ assessed by endoscopic indices has an integral role in the clinical treatment of patients with IBD. To date, many endoscopic indices of IBD have been introduced. However, most of them have not been validated for various reasons, such as the complexity of their parameters and inter-observer variations. Because of this, a chronological review of endoscopic indices is needed for managing IBD and for developing novel and efficient endoscopic indices.

\section{CHRONOLOGICAL APPROACH TO ENDOSCOPIC INDICES IN UC}

\section{Truelove and Witts Endoscopy Index - 1955}

In 1955, the first description of endoscopic features in UC was reported by Truelove and Witts. ${ }^{8}$ They suggested the outcomes of a randomized controlled trial of hydrocortisone therapy in active UC. In their study, they suggested a modality for estimating disease activity and named this scoring system as the Truelove and Witts Severity Index. It is composed of six variables: number of stools per day; blood in stools; temperature; pulse; hemoglobin level; and erythrocyte sedimentation rate. In addition, sigmoidoscopic findings were obtained at the end of the treatment and were classified as normal or near normal (slight hyperemia or slight granularity), improved, unchanged, or worse. Although the Truelove and Witts Endoscopy Index is a meaningful instrument for estimating the outcomes of medical treatment, it has not been used extensively because it is not a quantitative scoring modality.

\section{Baron Index - 1964}

In 1964, Baron et al. conducted a study on the inter-observer variability in describing the lesions of the rectum and sigmoid mucosa using a rigid sigmoidoscope in patients with UC. ${ }^{9}$ The objective of their study was to describe the mucosal appearances noted on sigmoidoscopy. Patients were divided into two groups. In the first group, the variables used for the evaluation of the mucosal layer included the color, deep vessels, superficial vessels, moisture, friability, granularity, distensibility, polyp, ulcer, and lumen (blood, mucous, and feces). In the second group, different variables were used in each patient for evaluating the mucosal layer (vessels, spontaneous bleeding ahead of the instrument, touch bleeding, granularity, mucosal surface, ulceration, and rectal valves) and grading severity based on the severity of bleeding (normal, abnormal but not hemorrhagic, moderately hemorrhagic, and severely hemorrhagic). To reduce inter-observer differences, the observers selected the variables from the two groups with the highest agreement. Although this index presented an agreement regarding specific findings of the mucosal appearances, it has not been validated owing to the complexity of its use.

\section{Powell-Tuck Index - 1982}

In 1982, Powell-Tuck et al. reported sigmoidoscopic findings that correlated with clinical disease activity. ${ }^{10}$ They conducted regular sigmoidoscopies when they performed a controlled trial of oral prednisolone as a therapy for patients with active UC. They compared the relationships between clinical signs and sigmoidoscopic appearances and categorized mucosal findings into non-hemorrhagic and hemorrhagic (bleeding on light touch and spontaneous bleeding). The study provided a clinically meaningful subdivision of hemorrhagic mucosa (bled spontaneously/bled on light touching or scraping).

\section{Sutherland Index - 1987}

In 1987, Sutherland et al. presented an assessment index of mucosal appearance. ${ }^{11}$ They used this during a placebo-controlled trial of mesalamine enemas in patients with distal UC. ${ }^{11}$ Mucosal appearances were described as normal, mild friability, moderate friability, exudation, or spontaneous bleeding. The authors insisted that the mucosal appearance assessment index in their study correlated to disease activity.

\section{Mayo Clinic Index: Endoscopic Sub-Score - 1987}

In 1987, Schroeder et al. presented the outcomes of a delayed-release oral form of mesalamine for therapy in patients with active UC. ${ }^{12}$ The authors presented a modality for measuring disease activity, namely, the Mayo Endoscopic Score (MES). The MES consists of four descriptors: stool frequency, rectal bleeding, findings of flexible sigmoidoscopy, and physician global assessment. They defined this endoscopic subscore using a four-point scale for grading (Fig. 1). To date, the MES has been the most widely used index in clinical trials; however, it has not been formally validated yet. Nevertheless, it has produced good consistency among reports. ${ }^{13}$

\section{Rachmilewitz Index - 1988}

In 1988, Rachmilewitz reported meaningful results on the use of coated mesalamine and sulfasalazine for therapy in patients with active UC. ${ }^{14}$ In the trial, the authors suggested a scoring system of symptoms and endoscopic findings, which includes seven descriptors: number of stools, blood in stools, investigators' global assessment of the symptomatic state, abdominal pain or cramps, fever, extra-intestinal manifestations, 

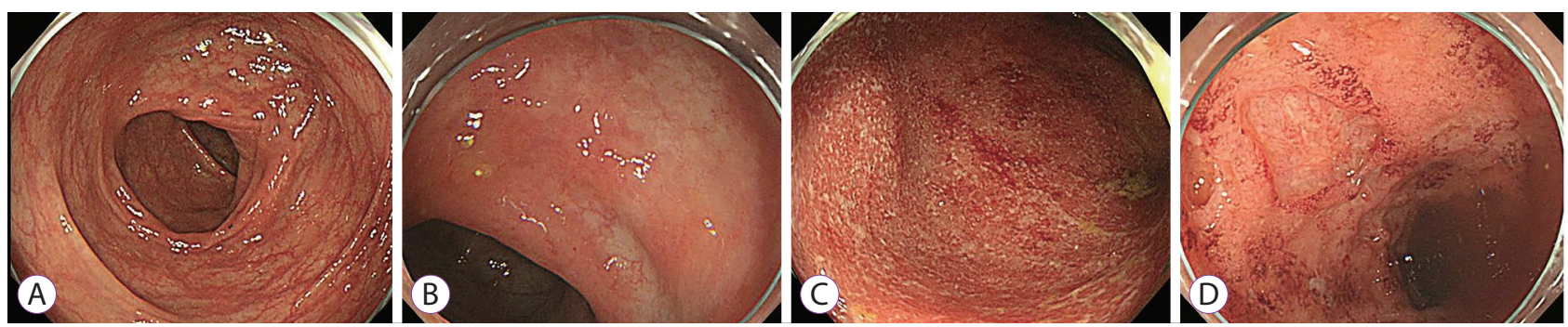

Fig. 1. Endoscopic images according to the Mayo Endoscopic Score. (A) Grade 0 (normal or inactive disease), (B) grade 1 (mild disease), (C) grade 2 (moderate disease), and (D) grade 3 (severe disease).

and laboratory findings. Further, the Rachmilewitz index uses four descriptors (granulation scattering reflected light, vascular pattern, vulnerability for the mucosa, and mucosal change). An endoscopic index of $0-4$ indicates endoscopic healing. However, this modality has not been validated yet.

\section{Modified Baron Index - 2005}

In 2005, Feagan et al. presented the outcomes of a placebo-controlled trial of anti- $\alpha 4 \beta 7$ integrin antibody for therapy of severe UC. ${ }^{15,16}$ In these reports, the authors described endoscopic activity using the Modified Baron Index (range, $0-4$; normal mucosa [0], granular mucosa with an abnormal vascular pattern [1], friable mucosa [2], microulceration with spontaneous bleeding [3], and gross ulceration [4]). A Modified Baron Index of 0 indicates endoscopic resolution. Additionally, endoscopic estimation of treatment efficacy was considered on the basis of an increasing Modified Baron Index of at least 2 from the initial score. This index has already been validated.

\section{Endoscopic Activity Index - 2010}

In 2010, Naganuma et al. presented an index of endoscopic activity used for selecting therapeutic agents in patients with severe and active UC. ${ }^{17}$ It is known as the Endoscopic Activity Index (EAI). The score has six descriptors, and scores of 0 to $2 / 3$ were assigned on the basis of the severity of the descriptor, with a higher score indicating a more severe disease status. The results obtained from applying the EAI in colonoscopies in patients with UC were correlated with those obtained from the Matts' classification or Rachmilewitz index. There was a wide variation in the EAIs in patients with the highest Matts' classification or Rachmilewitz Index; it was suggested that the EAI could provide a more precise grade of severe diseases. The authors insisted that the EAI was precisely correlated with other endoscopic and clinical indices, derived from their statistical analysis. However, because various descriptors are presented, inter-observer variations might occur.

\section{UC Endoscopic Index of Severity - 2012}

In 2012, Travis et al. introduced a novel modality to measure the endoscopic severity of UC, i.e., UC Endoscopic Index of Severity (UCEIS). ${ }^{18,19}$ The UCEIS was developed from a two-phase study using a library of 670 video sigmoidoscopies from patients with Mayo Clinic scores ranging from 0 to 11, added by 10 videos from five individuals without $\mathrm{UC}$ and five hospitalized patients with acute severe UC. In phase 1, each of 10 investigators estimated 16 of 24 videos to evaluate the agreements on the Baron Index with a central reader and agreed on the definitions of 10 endoscopic descriptors. In phase 2, each of 30 different investigators rated 25 of 60 different videos for the items and assessed the overall severity on a 0-100-point visual analog scale (VAS). An index that presents the overall endoscopic disease status evaluated on the VAS was then developed. This index, which is described using endoscopic appearances, including vascular pattern scored 1 to 3 , bleeding scored 1 to 4 , and erosions/ulcers scored 1 to 4, was divided into different levels of severity (Fig. 2). The UCEIS has three descriptors (vascular pattern, bleeding, and erosions and ulcers); however, this was sufficient to serve as an index considering disease activity related to UC. Although the UCEIS precisely predicted the severity of UC, the reliability and validity should be confirmed. Additionally, as with the previous endoscopic indices, it originates from the gross estimation of the involved colon segment and does not consider the microscopic involvement of UC. Therefore, it cannot be used to confirm partial MH. However, the UCEIS is a simple and reliable modality compared with the previous indices.

\section{UC Colonoscopic Index of Severity - 2013}

In 2013, Samuel et al. from the Mayo Clinic introduced a full colonoscopy severity index, UC Colonoscopic Index of Severity (UCCIS). ${ }^{20}$ Colonoscopy videos from 51 patients were evaluated by seven gastroenterologists and edited into video clips characterizing five segments of the colorectum. Absence or presence was scored on the basis of edema, erythema, stricture, change in the haustral folds, rigidity, and pseudopolyps. The vascular pattern, granularity, ulceration, and bleeding 

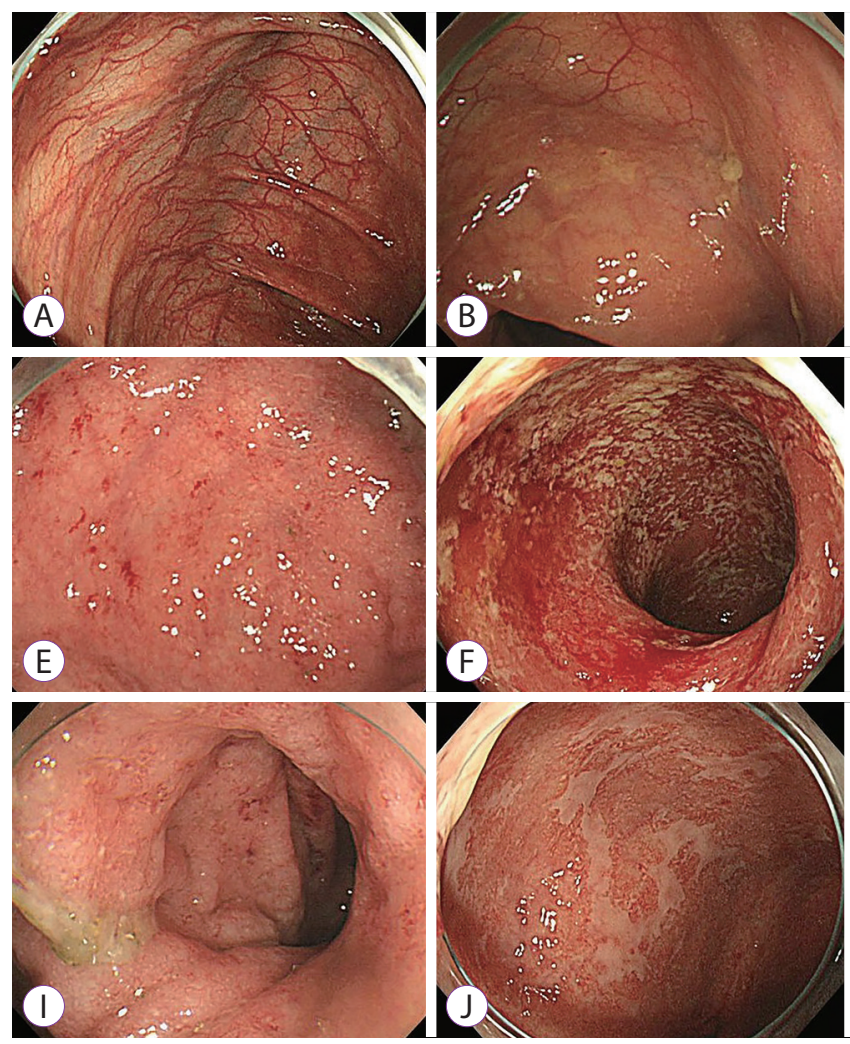
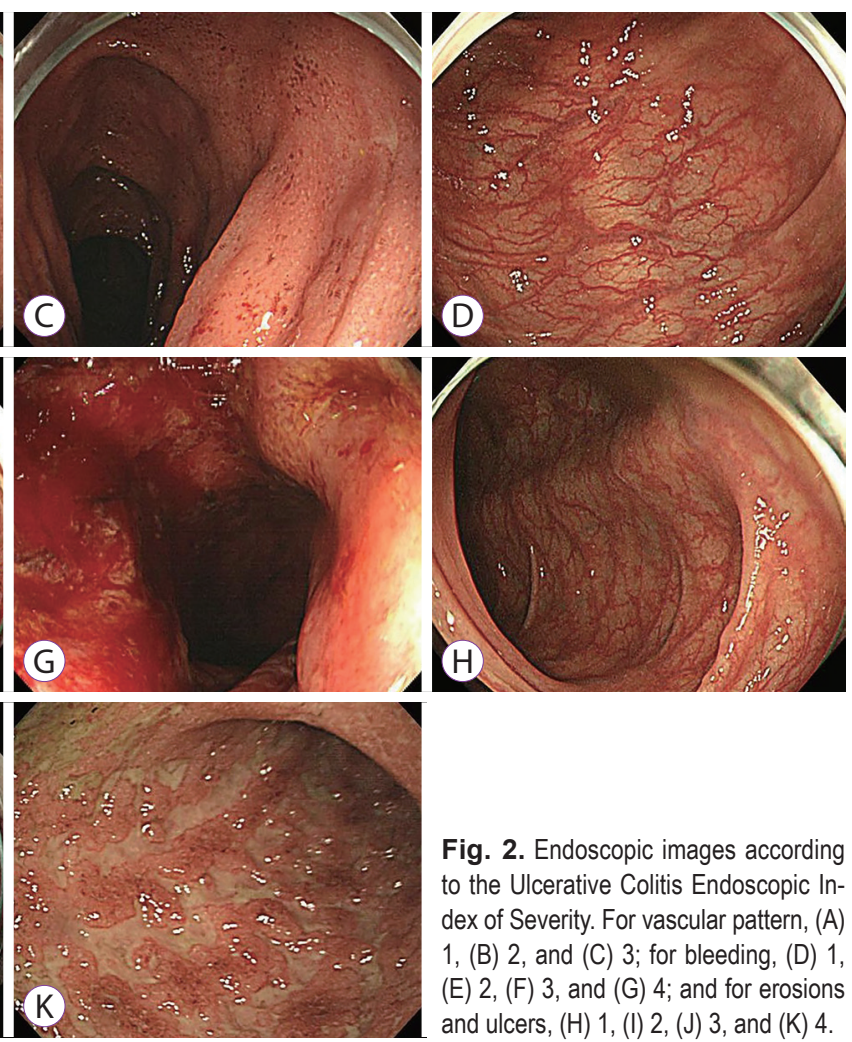

,

Fig. 2. Endoscopic images according to the Ulcerative Colitis Endoscopic Index of Severity. For vascular pattern, (A) 1, (B) 2, and (C) 3; for bleeding, (D) 1, (E) 2, (F) 3, and (G) 4; and for erosions and ulcers, (H) 1, (I) 2, (J) 3, and (K) 4. friability were scored between 0 and 4 for specific parameters. The global measurement of endoscopic severity was then rated on a four-point scale and a 10-cm VAS. The UCCIS was derived from these, and this index is composed of four different descriptors (granularity, vascular pattern, ulceration, and bleeding friability), all of which are summed from the five segments (rectum and sigmoid, descending, transverse, and ascending colon). The index had been validated with a high inter-observer agreement. ${ }^{20}$ However, there is a complexity in obtaining the final score. In this regard, although it is an accurate index, the UCCIS might not be simple enough to be used in clinical practice. Additionally, it has the inability to provide information on the histological status.

\section{Modified MES - 2015}

The MES is a widely known and used endoscopic index in clinical trials and practices. However, there was a need for a more precise measurement of endoscopic indices in UC that considers the severity and involvement of mucosal change on the basis of the MES. In 2015, Lobatón et al. introduced a novel index to estimate the segment involvement and endoscopic severity in patients with UC; this was conferred as the modified MES (MMES). ${ }^{21}$ Dividing the colon into five segments, the MES was obtained from each segment with a given modified score. This score is then calculated as the maximal extent of the disease and categorized by the number of segments with an active disease to obtain the MMES. The MMES includes the maximal extent of the involved segment; in cases of proctitis/left-sided colitis, sigmoidoscopy is usually sufficient for measurement. In addition, the MMES can be easily used for UC because it combines the disease status of the MES and a score correlated with the clinical and histological status. However, the MMES has some weak points. The endoscopies were not video-recorded; because of this, the inter-observer agreement was not measured and has yet to be validated.

\section{Summary}

In 1955, Truelove and Witts first commented on the mucosal status as a measurement of disease activity using rigid sigmoidoscopy in a placebo-controlled trial of cortisone for UC. The prototype endoscopic indices were introduced to estimate the endoscopic status of the disease but not the involved segment in UC. These indices include the Baron Index, Powell-Tuck Index, Sutherland Index, MES, and Rachmilewitz Index. Most of their endoscopic descriptors have a similarity. The MES is simple and widely used in clinical trials and practices for estimating the effectiveness of treatment. However, none of the endoscopic indices mentioned previously have been validated. To date, the UCEIS has been suggested to originate from grading systems including endoscopic findings. 
The UCEIS, in addition to the previous endoscopic indices, is based on the endoscopic evaluation of the disease extent and does not consider the histologic severity of the disease. To date, the UCCIS is the only endoscopic index covering all the colonic segments. This is measured by four different variables. All of these descriptors are calculated from each of the five segments of the colorectum. The UCCIS has been validated with a high correlation among observers. Among these endoscopic indices, the MES is the most commonly used index in clinical trials and practices. However, the features of the different levels overlap in the MES, and this endoscopic index is ambiguous in describing endoscopic findings, which causes high inter-observer variations. The summarized descriptors of these endoscopic indices are presented in Table 1.

\section{CHRONOLOGICAL APPROACH OF ENDOSCOPIC INDICES IN CD}

\section{Endoscopic Index of Severity - 1989}

The CD Endoscopic Index of Severity (CDEIS) was introduced in 1989 by the French group GETAID. ${ }^{22}$ Two endoscopists viewed colonoscopies from 75 patients. One endoscopist conducted the procedure, whereas the other endoscopist observed. The sum of the score (range, $0-44$ ) was measured from a formula composed of the presence and extent of the ulcerated and inflamed mucosa and stenosis from different portions (Table 2, Fig. 3). The CDEIS has a high sensitivity for measuring mucosal lesions. In the CDEIS, a score of less than or equal to 7 indicates endoscopic resolution, and a score of

Table 1. Descriptors of Endoscopic Indices in Ulcerative Colitis

\begin{tabular}{|c|c|c|c|c|c|c|c|c|}
\hline Index & VP & B & $\mathrm{E} / \mathrm{U}$ & $\mathbf{F}$ & G & $\mathbf{H}$ & ME & Ex \\
\hline Truelove and Witts & & & & & $\bullet$ & $\bullet$ & & \\
\hline Baron & $\bullet$ & $\bullet$ & & $\bullet$ & & & & \\
\hline Powell-Tuck & & $\bullet$ & & & & & & \\
\hline Suntherland & & $\bullet$ & & - & & & & $\bullet$ \\
\hline Mayo & $\bullet$ & $\bullet$ & $\bullet$ & $\bullet$ & & $\bullet$ & & \\
\hline Rachmilewitz & $\bullet$ & & $\bullet$ & $\bullet$ & - & & & \\
\hline Modified Baron & $\bullet$ & $\bullet$ & $\bullet$ & $\bullet$ & $\bullet$ & & & \\
\hline Endoscopic Activity & & $\bullet$ & $\bullet$ & & & $\bullet$ & $\bullet$ & $\bullet$ \\
\hline UCEIS & $\bullet$ & $\bullet$ & $\bullet$ & & & & & \\
\hline
\end{tabular}

B, bleeding; E/U, erosion/ulceration; Ex, exudate; F, friability; G, granularity; H, hyperemia; ME, mucosal edema; UCEIS, Ulcerative Colitis Endoscopic Index of Severity; VP, vascular pattern.

Table 2. Example of the CDEIS Scoring Form

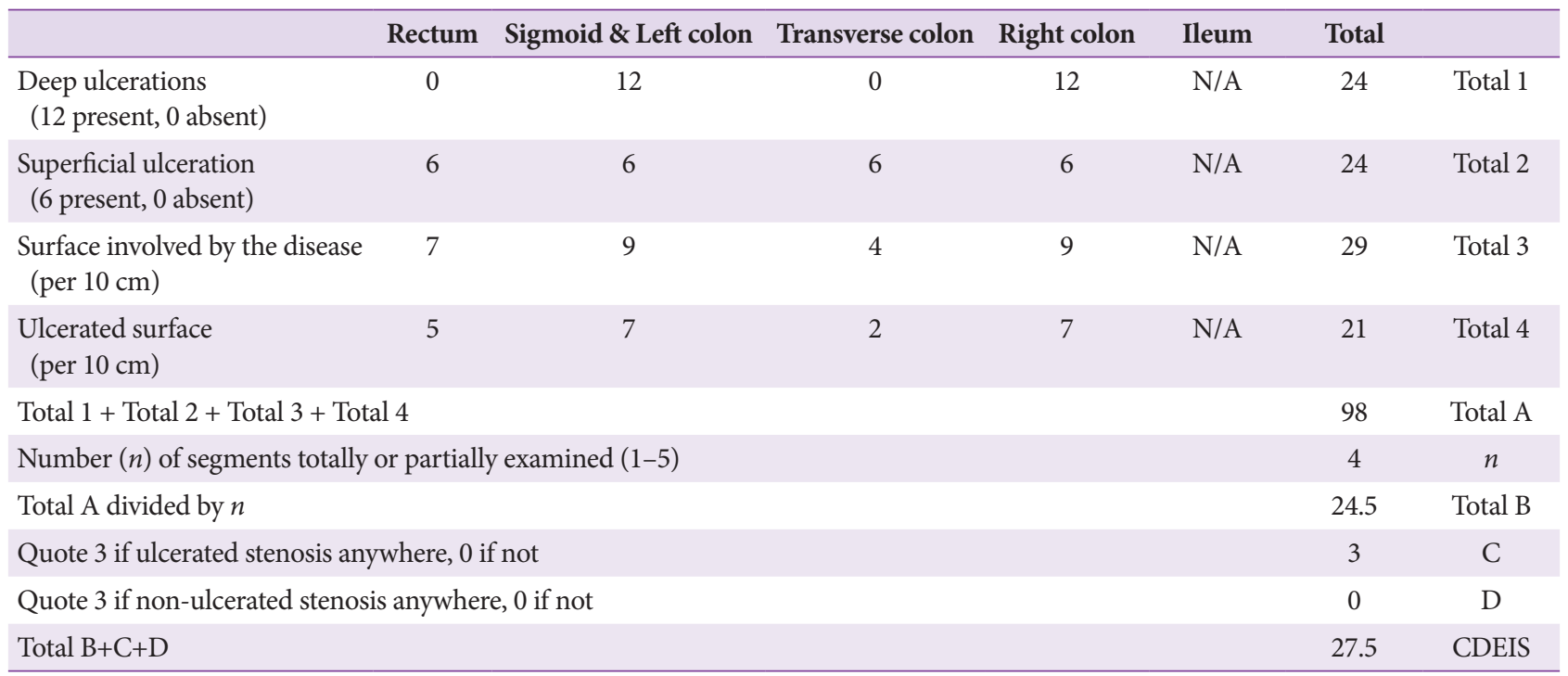

CDEIS, Crohn's Disease Endoscopic Index of Severity; N/A, not available. 

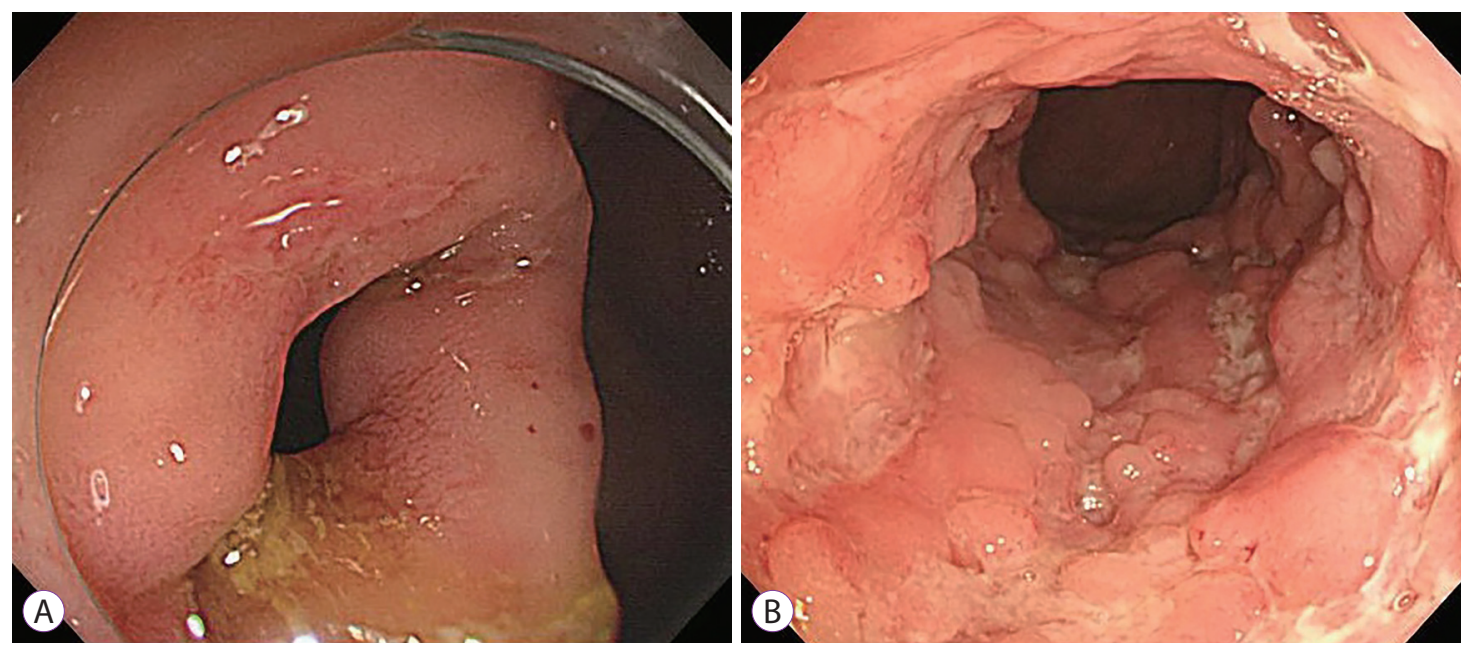

Fig. 3. Endoscopic images according to the Crohn's Disease Endoscopic Index of Severity. (A) Superficial ulceration and (B) deep ulceration.

less than or equal to 4 indicates complete endoscopic resolution. A score that decreases from the initial score by at least 4 or 5 points indicates an endoscopic response. The strength of the CDEIS is its high correlation with lesion severity and low inter-observer variability. A recent study demonstrated good agreement on using the CDEIS. ${ }^{23}$ Therefore, the CDEIS is considered a reproducible index. However, it is associated with complexity in usage and training needed to estimate the extent of diseased mucosal surfaces and to measure the depth of ulcerations.

\section{Simple Endoscopic Score for CD - 2004}

In 2004, the Simple Endoscopic Score for CD (SES-CD) was suggested to address the complexities of the CDEIS. ${ }^{24}$ The descriptors of the SES-CD were developed after reviewing the GETAID studies. From this effort, the SES-CD was found to include important and reproducible endoscopic characteristics of $\mathrm{CD}$. The SES-CD includes four variables from 0 to 3 in five segments of the ileum and colorectum. In this score, ulcers are categorized by size, and affected lesions with ulcerated surfaces and intestinal surfaces are classified according to extension (Figs. 4, 5). The total score is attained from the sum of all the descriptors in each segment (range, 0-56). Most importantly, the SES-CD correlates well with the CDEIS. In this regard, the SES-CD has a strength in clinical trials and practices. However, similar to the CDEIS, the SES-CD has some limitations. To date, there is no agreement on the cut-off values for defining the endoscopic efficacy of therapies and MH. Additionally, the SES-CD does not consider the number of examined portions.

\section{Rutgeerts Postoperative Endoscopic Index - 1984}

In cases of postoperative $\mathrm{CD}$, endoscopic findings were first described in $1984 .{ }^{25,26}$ In one of these reports, 114 patients underwent ileocolic resection. Less than 1 year after surgery,
29 of these 114 patients were examined, and $72 \%$ had recurrent $\mathrm{CD}$, which was mostly located at the neo-terminal ileum and ileocolic anastomosis site. Small aphthous ulcers in the ileocolic anastomosis site were the early endoscopic parameters of recurrence. At the first colonoscopy, ileal lesions were measured. The index was developed for grading endoscopic postoperative recurrence at the ileocolic anastomosis site. The Rutgeerts Postoperative Endoscopic Index is the only postoperative endoscopic scoring system. It has been used to assess the presence of endoscopic recurrence at the neo-terminal ileum. Therefore, it has become the standard index for estimation of $\mathrm{MH}$ in postoperative CD. However, this index has weak points in that it lacks validation and evaluation for inter-observer agreements.

\section{Summary}

The CDEIS is the standard index but is complex, whereas the SES-CD is a simplified version. The Rutgeerts Postoperative Endoscopic Index is used for estimating the risk of recurrence after ileocolic resection due to $\mathrm{CD}$. The CDEIS has strengths in that it is validated and has been used in several trials. This leads to early detection of endoscopic disease activity. Thus, it allows physicians to compare different endoscopic examinations. However, it is a complex scoring system that requires an effort to be summed and is not fit for use in clinical practice. The SES-CD simplified some CDEIS descriptors, and the results may be similar with those of the CDEIS items. However, this score is still complex, needs time to be calculated, and is not easy to use in clinical practice. The Rutgeerts Postoperative Endoscopic Index defines the severity of endoscopic disease recurrence at the anastomosis site postoperatively. It has some strengths, such as popularity and ease of use in routine practice. However, there are agreement concerns, and the index has not been validated yet. 

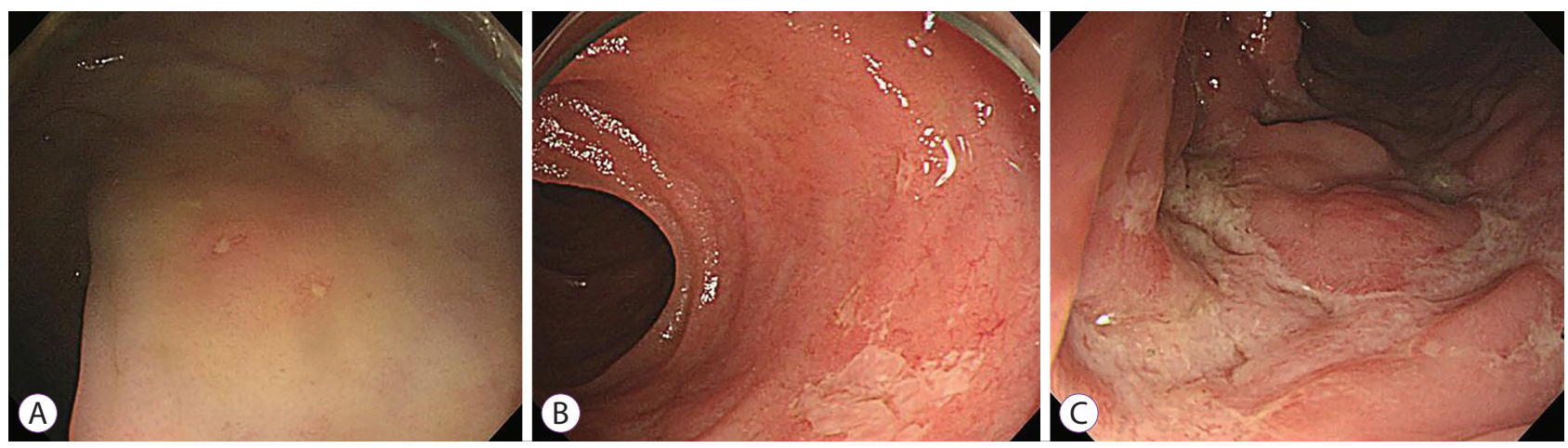

Fig. 4. Endoscopic images according to the Simple Endoscopic Score for Crohn's Disease. For the size of ulcers, (A) aphthous ulcers, (B) large ulcers, and (C) very large ulcers.
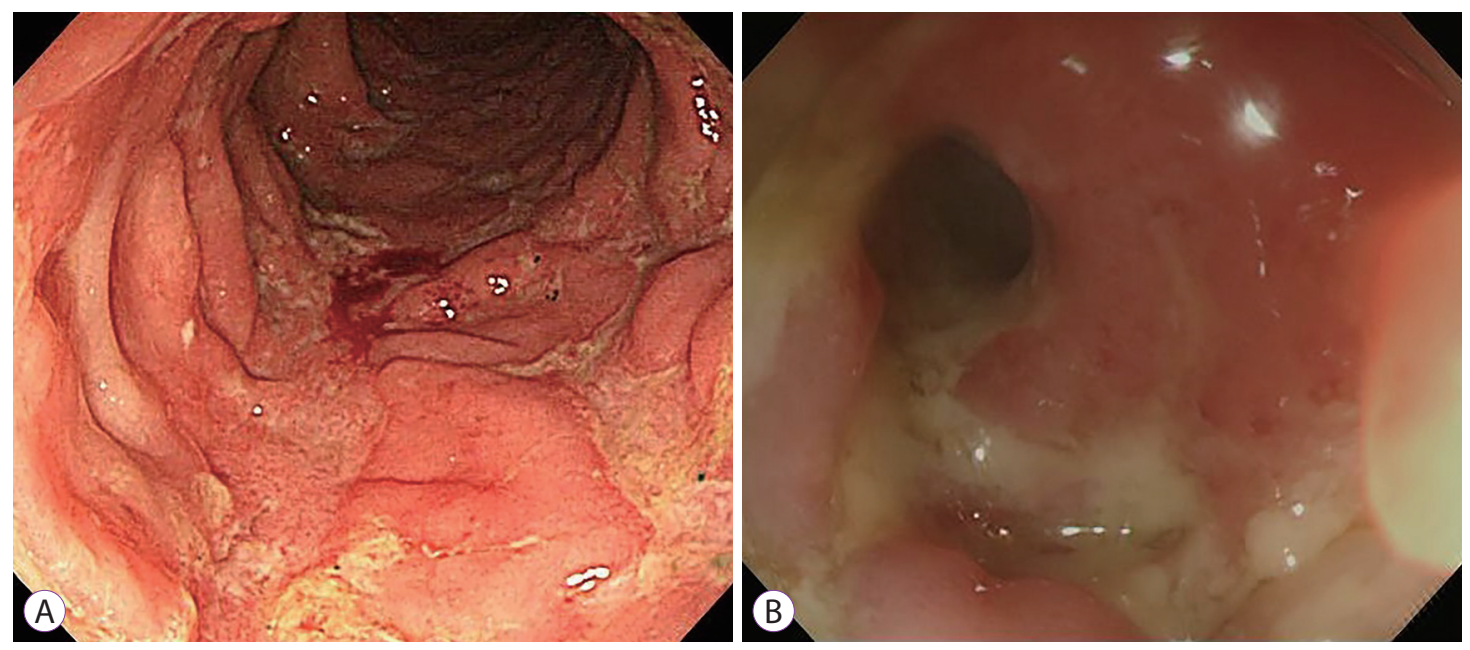

Fig. 5. Endoscopic images according to the Crohn's Disease Endoscopic Index of Severity (CDEIS) and Simple Endoscopic Score for Crohn's Disease (SES-CD). For ulcerated and affected surfaces, (A) CDEIS (score 10.0) and SES-CD (score 3.0) and for ulcerated impassable stenosis, (B) CDEIS (score 3.0) and SES-CD (score 3.0).

\section{CONCLUSIONS}

Performing endoscopy frequently and regularly may yield problems, including complaints of discomfort and low patient compliance. Although endoscopy is considered a lowrisk procedure, endoscopic procedures sometimes carry risks, such as perforation and bleeding. However, colonoscopy has a integral role in diagnosing IBD. ${ }^{27}$ Furthermore, endoscopic indices, as estimated by endoscopy, are needed for assessing and guiding treatment in patients with IBD; many endoscopic indices have been introduced and used in clinical trials and practices (Fig. 6). In this regard, more practical and reliable endoscopic indices should be introduced. Although there are many endoscopic indices, they present a low validation rate owing to the complexity of the scoring system and high variations. In future research, it is important for endoscopic indices to have simplicity in variables, reliability, validity, and responsiveness. To improve the evaluation of patients with

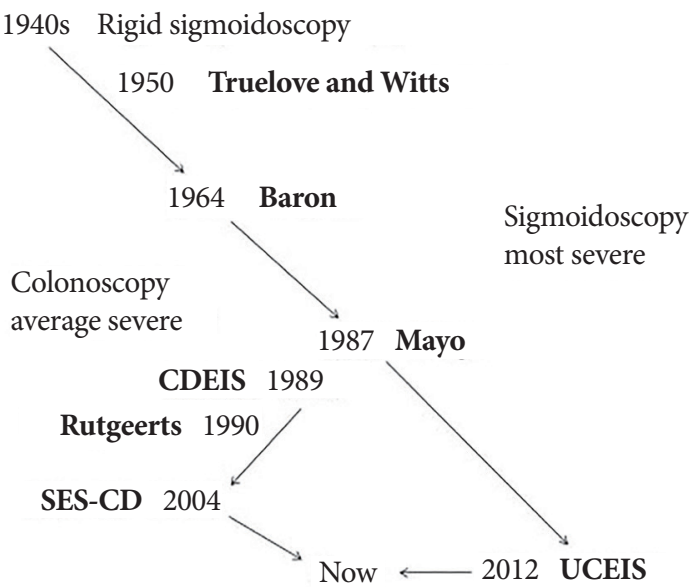

Fig. 6. Chronicle of endoscopic indices in inflammatory bowel disease. CDEIS, Crohn's Disease Endoscopic Index of Severity; SES-CD, Simple Endoscopic Score for Crohn's Disease; UCEIS, Ulcerative Colitis Endoscopic Index of Severity. 
IBD, endoscopic indices should present the histologic status of the intestinal mucosa and $\mathrm{MH}$.

\section{Conflicts of Interest}

The authors have no financial conflicts of interest.

\section{REFERENCES}

1. Neurath MF, Travis SP. Mucosal healing in inflammatory bowel diseases: a systematic review. Gut 2012;61:1619-1635.

2. Frøslie KF, Jahnsen J, Moum BA, Vatn MH. Mucosal healing in inflammatory bowel disease: results from a Norwegian population-based cohort. Gastroenterology 2007;133:412-422.

3. Feagan BG, Lémann M, Befrits R, et al. Recommendations for the treatment of Crohn's disease with tumor necrosis factor antagonists: an expert consensus report. Inflamm Bowel Dis 2012;18:152-160.

4. Annese V, Daperno M, Rutter MD, et al. European evidence based consensus for endoscopy in inflammatory bowel disease. J Crohns Colitis 2013;7:982-1018.

5. Dave M, Loftus EV Jr. Mucosal healing in inflammatory bowel disease-a true paradigm of success? Gastroenterol Hepatol (N Y) 2012;8:29-38.

6. Ardizzone S, Cassinotti A, Duca P, et al. Mucosal healing predicts late outcomes after the first course of corticosteroids for newly diagnosed ulcerative colitis. Clin Gastroenterol Hepatol 2011;9:483-489.e3.

7. Peyrin-Biroulet L, Ferrante M, Magro F, et al. Results from the 2nd Scientific Workshop of the ECCO (I): impact of mucosal healing on the course of inflammatory bowel disease. J Crohns Colitis 2011;5:477-483.

8. Truelove SC, Witts LJ. Cortisone in ulcerative colitis; final report on a therapeutic trial. Br Med J 1955;2:1041-1048.

9. Baron JH, Connell AM, Lennard-Jones JE. Variation between observers in describing mucosal appearances in proctocolitis. Br Med J 1964;1:8992.

10. Powell-Tuck J, Day DW, Buckell NA, Wadsworth J, Lennard-Jones JE. Correlations between defined sigmoidoscopic appearances and other measures of disease activity in ulcerative colitis. Dig Dis Sci 1982;27:533537.

11. Sutherland LR, Martin F, Greer S, et al. 5-Aminosalicylic acid enema in the treatment of distal ulcerative colitis, proctosigmoiditis, and proctitis. Gastroenterology 1987;92:1894-1898.

12. Schroeder KW, Tremaine WJ, Ilstrup DM. Coated oral 5-aminosalicylic acid therapy for mildly to moderately active ulcerative colitis. A randomized study. N Engl J Med 1987;317:1625-1629.
13. Osada T, Ohkusa T, Yokoyama T, et al. Comparison of several activity indices for the evaluation of endoscopic activity in UC: inter- and intraobserver consistency. Inflamm Bowel Dis 2010;16:192-197.

14. Rachmilewitz D. Coated mesalazine (5-aminosalicylic acid) versus sulphasalazine in the treatment of active ulcerative colitis: a randomised trial. BMJ 1989;298:82-86.

15. Feagan BG, Greenberg GR, Wild G, et al. Treatment of ulcerative colitis with a humanized antibody to the alpha4beta7 integrin. N Engl J Med 2005;352:2499-2507.

16. Feagan BG, Sandborn WJ, D'Haens G, et al. The role of centralized reading of endoscopy in a randomized controlled trial of mesalamine for ulcerative colitis. Gastroenterology 2013;145:149-157.e2.

17. Naganuma $M$, Ichikawa $H$, Inoue $\mathrm{N}$, et al. Novel endoscopic activity index is useful for choosing treatment in severe active ulcerative colitis patients. J Gastroenterol 2010;45:936-943.

18. Travis SP, Schnell D, Krzeski P, et al. Developing an instrument to assess the endoscopic severity of ulcerative colitis: the Ulcerative Colitis Endoscopic Index of Severity (UCEIS). Gut 2012;61:535-542.

19. Travis SP, Schnell D, Krzeski P, et al. Reliability and initial validation of the ulcerative colitis endoscopic index of severity. Gastroenterology 2013;145:987-995

20. Samuel S, Bruining DH, Loftus EV Jr, et al. Validation of the ulcerative colitis colonoscopic index of severity and its correlation with disease activity measures. Clin Gastroenterol Hepatol 2013;11:49-54.e1.

21. Lobatón T, Bessissow T, De Hertogh G, et al. The Modified Mayo Endoscopic Score (MMES): a new index for the assessment of extension and severity of endoscopic activity in ulcerative colitis patients. J Crohns Colitis 2015;9:846-852.

22. Mary JY, Modigliani R. Development and validation of an endoscopic index of the severity for Crohn's disease: a prospective multicentre study. Groupe d'Etudes Therapeutiques des Affections Inflammatoires du Tube Digestif (GETAID). Gut 1989;30:983-989.

23. Daperno $\mathrm{M}$, Comberlato $\mathrm{M}$, Bossa $\mathrm{F}$, et al. Inter-observer agreement in endoscopic scoring systems: preliminary report of an ongoing study from the Italian Group for Inflammatory Bowel Disease (IG-IBD). Dig Liver Dis 2014;46:969-973.

24. Daperno M, D’Haens G, Van Assche G, et al. Development and validation of a new, simplified endoscopic activity score for Crohn's disease: the SES-CD. Gastrointest Endosc 2004;60:505-512.

25. Rutgeerts P, Geboes K, Vantrappen G, Kerremans R, Coenegrachts JL, Coremans G. Natural history of recurrent Crohn's disease at the ileocolonic anastomosis after curative surgery. Gut 1984;25:665-672.

26. Rutgeerts P, Geboes K, Vantrappen G, Beyls J, Kerremans R, Hiele M. Predictability of the postoperative course of Crohn's disease. Gastroenterology 1990;99:956-963.

27. Lee JM, Lee KM. Endoscopic diagnosis and differentiation of inflammatory bowel disease. Clin Endosc 2016;49:370-375. 